\title{
Predictor and Prognosis of Procedural Rupture during Coil Embolization for Unruptured Intracranial Aneurysm
}

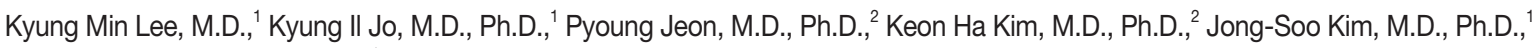 \\ Seung-Chyul Hong, M.D., Ph.D. ${ }^{1}$ \\ Departments of Neurosurgery, ${ }^{1}$ Radiology, ${ }^{2}$ Samsung Medical Center, Sungkyunkwan University School of Medicine, Seoul, Korea
}

\begin{abstract}
Objective : The objectives of this study was to determine the incidence and outcomes of procedural rupture (PR) during coil embolization of unruptured intracranial aneurysm (UIA) and to explore potential risk factors.

Methods : This retrospective study evaluated 1038 patients treated with coil embolization between January 2001 and May 2013 in a single tertiary medical institute. PR was defined as evidence of rupture during coil embolization or post procedural imaging. The patient's medical records were reviewed including procedure description, image findings and clinical outcomes.

Results : Twelve of 1038 (1.1\%) patients showed PR. Points and time of rupture were parent artery rupture during stent delivery ( $\mathrm{n}=2)$, aneurysm rupture during filling stage $(n=9)$ and unknown $(n=1)$. Two parent artery rupture and one aneurysm neck rupture showed poor clinical outcomes [modified Rankin Scale (mRs) >2] Nine aneurysm dome rupture cases showed favorable outcomes (mRS $\leq 2)$. Location (anterior cerebral artery) of aneurysm was associated with high procedural rupture rate $(p<0.05)$.

Conclusion : The clinical course of a patientwith procedural aneurysm rupture during filling stage seemed benign. Parent artery and aneurysm neck rupture seemed relatively urgent, serious and life threatening. Although the permanent morbidity rate was low, clinicians should pay attention to prevent PR, especially when confronting the anterior cerebral artery aneurysm.
\end{abstract}

Key Words : Procedural rupture $\cdot$ Intracranial aneurysm $\cdot$ Endovascular $\cdot$ Unruptured.

\section{INTRODUCTION}

The prevalence of intracranial unruptured aneurysm has been reported as $3.2 \%$ in the general population ${ }^{21)}$. Although previously reported annual rupture rates are $l_{0}{ }^{22)}$, preventive treatments are widely accepted and performed. When coil embolization was approved as a treatment for intracranial aneurysm surgical treatment was the only option ${ }^{3)}$. Over the next 2 decades various coil embolization techniques have been applied worldwide.

Coil embolization has some benefits in treating an intracranial aneurysm. It can be performed without major craniotomy. Therefore it is less influenced by a patient's general medical condition and can easily meet the patient's preference. The symptomatic complication rate was not inferior to surgical treatment $^{4)}$. A higher thromboembolic complication rate has been described but most thromobembolic events are asymptomatic and does not usually influence cognitive functions ${ }^{10,12,18)}$.
Although procedural rupture (PR) accounts for a small percentage (0.5-4\%) of complications during coil embolization but can be disastrous with severe morbidity or mortality $(20-40 \%)^{2,17)}$. However, conclusions are hindered by the relatively small study populations and heterogeneous nature of the patients with ruptured and unruptured intracranial aneurysms ${ }^{2,17,23)}$. PR during coil embolization for aneurysms with subarachnoid hemorrhage (SAH) cannot demonstrate the outcomes of procedural rupture due to the renowned poor outcome of the preexisting $\mathrm{SAH}^{15)}$.

We reviewed more than 1000 consecutive cases of coil embolizations for unruptured intracranial aneurysms performed at our institution over the past 12 years to identify the incidence and outcomes of PR and to explore potential risk factors of PR.

\section{MATERIALS AND METHODS}

We retrospectively reviewed all unruptured saccular aneurysm cases treated with coil embolization in a tertiary academic

- Received : June 29, $2015 \cdot$ Revised : September 11, $2015 \cdot$ Accepted : October 28, 2015

- Address for reprints : Seung-Chyul Hong, M.D.

Department of Neurosurgery, Samsung Medical Center, Sungkyunkwan University School of Medicine, 81 Irwon-ro, Gangnam-gu, Seoul 06357, Korea

Tel : +82-2-3410-3499, Fax : +82-2-3410-0048, E-mail : schongns.hong@samsung.com

- This is an Open Access article distributed under the terms of the Creative Commons Attribution Non-Commercial License (http://creativecommons.org/licenses/by-nc/3.0) which permits unrestricted non-commercial use, distribution, and reproduction in any medium, provided the original work is properly cited. 
hospital between January 2001and May 2013. This consisted of 1086 aneurysm coil treatment procedures in 1038 patients. Exclusion criteria were ruptured aneurysms, mycotic aneurysms, and dissecting aneurysms. Data collected from medical records and imaging findings included age, sex, size, and location of aneurysm, type of endovascular procedure, type of rupture, postprocedural management, and clinical status of PR patients until the latest follow-up. Demographic characteristics were documented and video file records were reviewed to identify PR during procedure and/or postoperative imaging. The aneurysm size was documented from the operative notes during the coil embolization and/or procedural imaging. Aneurysm locations were categorized as anterior cerebral artery, middle cerebral artery, distal internal cerebral artery, posterior circulation, and multiple according to their location.

PR was defined as angiographic visualization of the extravascular leakage of contrast medium or identifiable SAH on post procedural computed tomography (CT) and/or magnetic resonance imaging (MRI). In our strategy, We performed immediate postprocedural CT and MRI according to physician's or patient's preference or any neurologic deterioration. PR was investigated with respect to timing, location of rupture, and clinical outcomes. PR was categorized as procedural parent artery rupture (PPAR) and procedural aneurysmal rupture (PAR) according to rupture site (Fig. 1). In one case where the rupture site was not identified during procedure, hemorrhage was assumed to be PAR. Out of concern that PPAR might result from a mechanism other than PAR, statistical analyses were performed regarding for PAR solitarily. Modified Rankin Scale (mRS) was used to assess clinical outcomes after PR.

\section{Procedure}

All aneurysm coiling procedures were performed using a commercially available biplane angiographic unit equipped with an image-intensifier. Rotational angiography, followed by three-dimensional image reconstruction by volume rendering, was performed before embolization in all patients. Final angiograms in the frontal and lateral projections and working projections were acquired at the end of each procedure in order to rule out distal branch occlusions. All patients were treated while

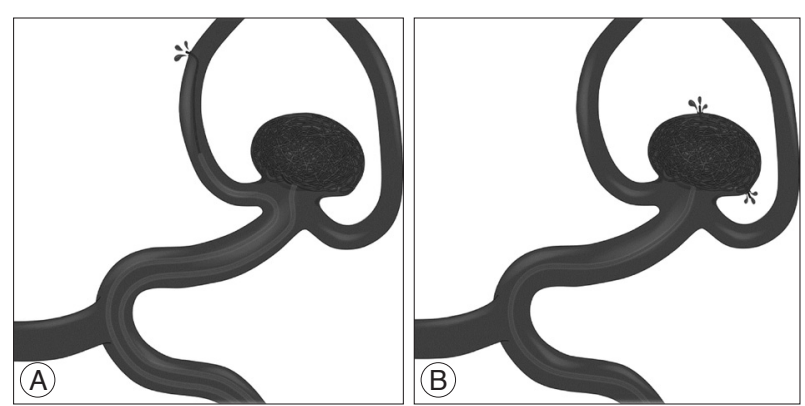

Fig. 1. Schematic images demonstrating type of rupture in this study. Types of rupture were categorized into two types-procedural parent artery rupture $(A)$, procedural aneurysm (dome or neck) rupture $(B)$ during coil embolization. under general anesthesia and systemic heparinization was administered after placement of the femoral introducer sheath.

In line with our embolization protocol, $3000 \mathrm{IU} / 60 \mathrm{~kg}$ of heparin was administered as an intravenous bolus injection, followed by an additional $1000 \mathrm{IU}$ with routine activated coagulation and thromboplastin time check. After embolization, heparin injection was continued for 24 hours in patients treated with stent assisted coil embolization and for selected cases without stent assisted coil embolization. All aneurysm embolizations were performed using commercially available detachable coils including hydrogel-coated coils, Guglielmi detachable coils, or other bare platinum coils. Each femoral puncture site was closed using a commercially available closing device. Balloon, stent, or catheter assisted coil embolization has been chosen for coil embolization of aneurysms with an unfavorable configuration. Procedure related factors such as embolization methods (conventional or adjunctive device, such as balloon or stent assisted technique) were also included.

\section{Statistical analyses}

Univariate and multivariate statistical analysis was performed to explore the potential risk factors of PAR. Frequency comparison was performed using the Fisher exact test. Multiple logistic regression test was used for multivariate analysis. Differences were considered significant at $p<0.05$. Statistical analysis was performed with SPSS 20.0 software (SPSS Inc., Chicago, IL, USA).

\section{RESULTS}

Among 1086/1038 saccular aneurysm/patients coil embolization, 34 (3.2\%) cases were retreated. Baseline characteristics of the patients are described in Table 1 . All data for each variable of interest were fulfilled. PR occurred in 12 patients (1.1\%). Rupture points of these patients were parent artery $(n=2)$, aneurysm itself $(n=9)$, and unknown rupture site $(n=1)$. Eleven PRs were identified during coil embolization and one PR was identified on a post-procedural CT. Two parent artery ruptures probably occurred during the stent delivery procedure (Fig. 2). Nine aneurysm ruptures occurred during filling stage after frame coil insertion. Among 10 cases of aneurysm rupture, eight occurred in the aneurysm dome area, one occurred in the aneurysm neck, and one was unknown (Table 2).

Additional coil insertion was performed in all cases with aneurysm rupture. Protamine sulfate was applied in the patients with aneurysm neck rupture because of procedural difficulties during additional coil insertion in the rupture point. Postprocedural CT of the Patient with aneurysm neck rupture showed extensive subarachnoid hemorrhage (SAH), intracerebral hemorrhage (ICH) and intraventricular hemorrhage (IVH). Except this patient, 8 out of 9 aneurysm rupture showed mild to moderate subarachnoid hemorrhage.

Contrast leakage of one parent artery rupture was stopped spontaneously with thrombus formation. Other parent artery 
rupture showed prolonged contrast leakage despite of protamine sulfate and proximal balloon occlusion. Postprocedural CT showed extensive ICH, IVH, and SAH in both patients.

Two patients with parent artery rupture and one with aneurysm neck rupture received subsequent craniectomy with hematoma removal but showed poor outcome at latest follow up. After 3, 19, and 39 months of follow-up, mRS of these patients were 5,4 , and 4 , respectively.

Among 8 patients who experienced aneurysm dome rupture during filling stage and one patient with unknown rupture site, 1 patient received a external ventricular drainage temporarily

Table 1. Baseline characteristics of the patients

\begin{tabular}{lc}
\multicolumn{1}{c}{ Characteristics } & $\begin{array}{c}\text { Value } \\
\text { (No. of procedural aneurysm rupture) }\end{array}$ \\
\hline Gender (male/female) & $312 / 726(2 / 8)$ \\
Age & $57.1 \pm 10.1$ (mean \pm standard deviation) \\
Location of aneurysm & \\
Distal ICA & $624(2)$ \\
ACA & $184(6)$ \\
MCA & $53(0)$ \\
Posterior circulation & $130(1)$ \\
Multiple & $47(1)$ \\
Coil technique & \\
Simple & $690(7)$ \\
Balloon assisted & $52(0)$ \\
Stent assisted & $296(3)$ \\
Aneurysm size & \\
$<10$ mm & $899(8)$ \\
$\geq 10$ mm & $139(2)$ \\
Retreatment & $34(0)$ \\
\hline
\end{tabular}

ICA : internal cerebral artery, ACA : anterior cerebral artery, MCA : middle cerebral artery and three patients received serial spinal tapping for the coexisting hydrocephalus. Nevertheless none of the patients required permanent shunting. After 34 months (median, range 4-59) of follow-up, morbidity or mortality were absent (mRS=0), except for one patient $(\mathrm{mRS}=2)$ complicated by subsequent ischemic insult on the parent artery.

Among 10 patients with aneurysm rupture, six were anterior cerebral artery aneurysms and the location of aneurysm was the only associated factor of PAR (anterior cerebral artery vs. others $=6 / 184$ vs. $4 / 854, p=0.003$ Fisher-exact test). Other variables such as age ( $<65$ vs. $\geq 65, p=0.500)$, sex $(p=0.382)$, aneurysm size ( $<10 \mathrm{~mm}$ vs. $\geq 10 \mathrm{~mm}, p=0.397)$ or usage of stent or

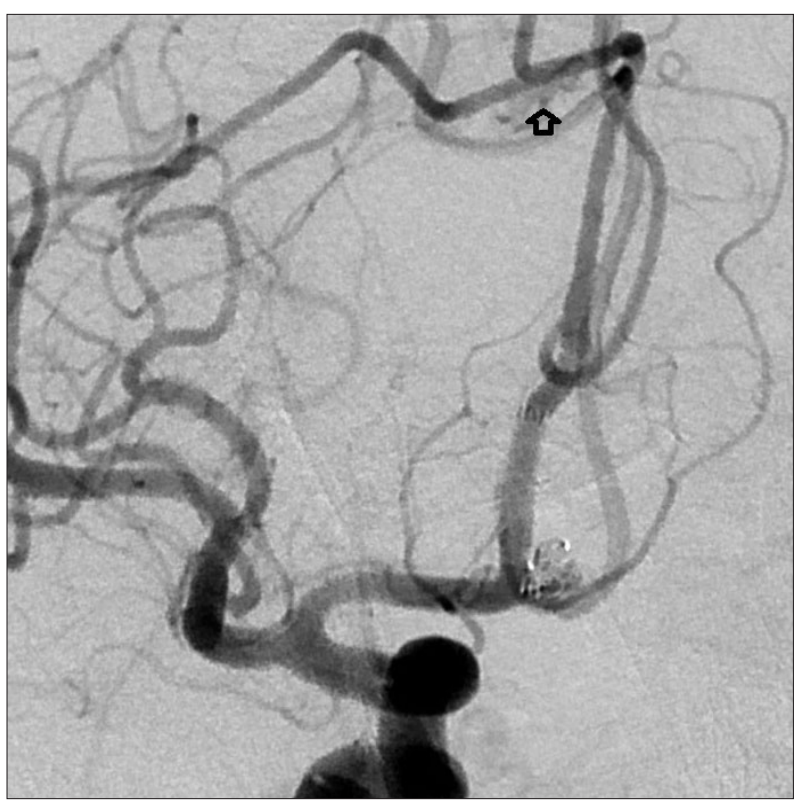

Fig. 2. Digital subtracted angiography showed contrast leakage (arrow) at distal anterior cerebral artery after stent deployment.

Table 2. Detailed information of the patients with procedural rupture

\begin{tabular}{|c|c|c|c|c|c|c|c|c|}
\hline & $\begin{array}{l}\text { Age (year)/ } \\
\text { sex }\end{array}$ & $\begin{array}{c}\text { Location } \\
\text { of aneurysm }\end{array}$ & $\begin{array}{l}\text { Size } \\
(\mathrm{mm})\end{array}$ & Technique & Rupture point & $\begin{array}{l}\text { Follow up periods } \\
\text { (months) }\end{array}$ & $\begin{array}{c}\mathrm{mRS} \text { at latest } \\
\text { follow up }\end{array}$ & Note \\
\hline 1 & $57 / \mathrm{F}$ & A-com & 5.1 & Simple & Dome & 121 & 2 & Subsequent infarct ACA \\
\hline 2 & $52 / \mathrm{F}$ & Basilar tip & 4.0 & Simple & Dome & 59 & 0 & Serial spinal tapping \\
\hline 3 & $57 / \mathrm{F}$ & A-com & 4.3 & Simple & Dome & 31 & 0 & $\mathrm{NC}$ \\
\hline 4 & $61 / \mathrm{F}$ & A-com & 4.3 & Simple & Dome & 38 & 0 & $\mathrm{NC}$ \\
\hline 5 & $62 / \mathrm{F}$ & A-com & 9.2 & Stent assisted & Parent artery & 39 & 4 & $\begin{array}{l}\text { Craniectomy } \\
\text { hematoma removal }\end{array}$ \\
\hline 6 & $65 / F$ & A-cho & 10.1 & Stent assisted & Dome & 37 & 0 & EVD \\
\hline 7 & $47 / \mathrm{F}$ & A-com & 3.9 & Simple & Dome & 36 & 0 & Serial spinal tapping \\
\hline 8 & $53 / \mathrm{M}$ & A-com & 3.8 & Simple & Neck & 25 & 5 & $\begin{array}{l}\text { Craniectomy } \\
\text { hematoma removal }\end{array}$ \\
\hline 9 & $74 / \mathrm{F}$ & A-com & 4.7 & Stent assisted & Parent artery & 20 & 4 & $\begin{array}{l}\text { Craniectomy } \\
\text { hematoma removal }\end{array}$ \\
\hline 10 & $78 / \mathrm{F}$ & A $2 / 3$ & 3.9 & Simple & Dome & 10 & 0 & $\mathrm{NC}$ \\
\hline 11 & $71 / \mathrm{F}$ & P-com & 10.2 & Stent assisted & Dome & 7 & 0 & Serial spinal tapping \\
\hline 12 & $53 / \mathrm{F}$ & $\begin{array}{l}\text { Ophthalmic } \\
\text { (bilateral) }\end{array}$ & 7.1 & Stent assisted & Unknown & 7 & 0 & $\mathrm{NC}$ \\
\hline
\end{tabular}

A-com : anterior communicating artery, A-cho : anterior choroidal artery, ACA : anterior cerebral artery, NC : no comment, EVD : external ventricular drainage 
Table 3. Uni- and multivariate analysis results for risk factor of aneurysmal rupture during coil embolization

\begin{tabular}{lccccc}
\hline & Univariate analysis & Multivariate analysis & Odds ratio & \multicolumn{2}{c}{ 95\% confidence interval } \\
\cline { 4 - 6 } & & & & Lower & Upper \\
\hline Male gender & 0.382 & 0.160 & 3.189 & 0.633 & 16.058 \\
Age $(\geq 65)$ & 0.500 & 0.883 & 1.110 & 0.277 & 4.445 \\
Size of aneurysm $(\mathrm{mm})(\geq 10$ vs. $<10)$ & 0.397 & 0.336 & 0.449 & 0.088 & 2.293 \\
Technique & & & & \\
Stent assisted vs. others & 0.572 & 0.379 & 0.507 & 0.112 & 2.299 \\
Aneurysm location & & & & 3.125 & 55.556 \\
ACA vs. Others & 0.003 & 0.000 & 13.333 & & \\
\hline
\end{tabular}

not $(p=0.572)$ did not show statistically significant difference. Multivariate analysis supported the results of univariate analysis. The ACA location was the only associated factor with procedural aneurysm rupture ( $p<0.001$; odds ratio, 13.333 ; $95 \% \mathrm{CI}$ 3.125-55.556). Aneurysm size did not show statistical significance in uni- and multivariate analyses in this study (Table 3).

\section{DISCUSSION}

In our case series eight aneurysm dome ruptures occurred during filling stage. Fortunately, all of these cases and unknown site leakage cases showed favorable outcomes with independency at the latest follow up. However one aneurysmal neck rupture showed poor outcome. This may reflect different in flow dynamics during coil embolization. Flow arrest identified by contrast stasis at the distal portion (dome area) of aneurysm from coil material is frequently identified during coil embolization $^{1,8)}$. Hwang et al. ${ }^{8)}$ reported that $73.1 \%$ of contrast stasis was located at the dome area. Rupture of these areas can be benign due to spontaneous thrombus formation. Thrombus formation can be facilitated by low flow rate and foreign material at the dome portion of aneurysm even after frame coil insertion ${ }^{16)}$. Therefore, extravasation might be minimal in $\mathrm{PR}$ at the dome area during the filling stage. However the neck portion of aneurysm can be considerably influenced by a parent artery flow rather than the dome portion by coil material, especially given a loosely packed aneurysm status ${ }^{11)}$. Technical difficulty of additional coil insertion conducted in the neck area may also be a reason of prolonged blood leakage. When PR occurs in a loosely packed neck position these factors may cause huge extravasation of blood and poor clinical outcomes.

Most previous studies on PR focused only on aneurysm rupture during coil embolization ${ }^{2,6,17}$. But our results show that parent artery injury can actually occur and seems to trigger more disastrous complications. Aneurysmal ruptures can be corrected in selected cases by an endovascular technique, such as additional coil insertion or temporary flow arrest using balloon ${ }^{17,23)}$. Distal parent artery perforation may be difficult to control through the endovascular technique in our experience and could result in poor clinical outcomes. Therefore excessive precaution is essential when approaching the distal angulated portion, especially when using adjunctive device. Development of a safer stent delivery system seems necessary in manipulating the distal vascular territory.

Interestingly, 8 of $12 \mathrm{PR}$, and 6 of $10 \mathrm{PAR}$ events occurred in ACA. This may reflect the difficult anatomical characteristics for endovascular coil embolization of the ACA aneurysm, such as unfavorable dome neck ratio and acute ICA-A1 angle ${ }^{5)}$. Previous studies on coil embolization for ACA aneurysm also demonstrated high risk of rupture ${ }^{20)}$. Additionally the surgical manipulation of this area remains challenging due to the fact of multiple vital perforating arteries and high risk of premature rupture ${ }^{7)}$, which makes it difficult to say if substitutional surgical method can be preferred. Also ACA located aneurysm had high risk of rupture even small sized aneurysm if left untreated ${ }^{9,14,19,22}$. To the best of our knowledge, there is no absolute guideline in treating ACA aneurysms. An interdisciplinary approach to ACA aneurysm is needed and a more careful procedure is required. Further prospective controlled studies comparing the outcomes between treatment modality and risk factors in treating ACA aneurysms could increase better understanding and may help determine appropriate treatment strategy.

All PARs occurred during the filling stage in this study. This finding reflects a technical limitation. During aneurysm selection and frame coil insertion, an experienced operator can see and predict the motion of microcatheters, microwires, and coil material. Detached coil materials could block the operator's view and the additional coil could be influenced by previously inserted coils, restricting prediction of the location and direction mode of action of additional coil which can be a cause of PAR.

Our study showed relatively favorable outcomes when comparing with recently published studies focusing on $\mathrm{PR}^{13,23)}$. One of the reasons might have resulted from the inclusion criteria of this study. We only included patients with unruptured aneurysms excluding ruptured aneurysms based on the hypothesis that a ruptured aneurysm could be anunfavorable candidate to elucidate the outcomes of PR. Since SAH itself can lead to morbidity in $25 \%$ of the patients ${ }^{15)}$.

The present study has several limitations that should be noted. In particular, it is limited by its retrospective nature. Not all of the patients received immediate postprocedural CT or MRI, which means that the patients with silent or mild symptomatic PR not identified during procedure could have been missed. Patients were treated with heterogeneous devices which can in- 
fluence our results. Other factors, such as vascular tortuosity, connective tissue disease and other medical conditions that possibly influence the risk of rupture and clinical outcomes were not considered in this study.

\section{CONCLUSION}

PR can result in a disastrous outcome in treating unruptured intracranial aneurysms. PPAR and rupture at the neck portion of the aneurysm seemed to result in poor clinical outcome, nevertheless PARs during the filling stage seems to have a relatively benign course. We should pay more attention when treating ACA aneurysms to avoid PR. Further studies regarding detailed risk factors and outcomes of endovascular treatment and clipping is required to improve our understanding and to set balanced interdisciplinary treatment strategies.

\section{References}

1. Abdihalim MM, Chaudry SA, Tariq N, Suri MF, Qureshi AI : The natural history of "contrast stasis" within aneurysm after embolization. J Vasc Interv Neurol 5 : 14-21, 2013

2. Elijovich L, Higashida RT, Lawton MT, Duckwiler G, Giannotta S, Johnston SC; Cerebral Aneurysm Rerupture After Treatment (CARAT) Investigators : predictors and outcomes of intraprocedural rupture in patients treated for ruptured intracranial aneurysms : the CARAT study. Stroke 39 : 1501-1506, 2008

3. Fernandez Zubillaga A, Guglielmi G, Viñuela F, Duckwiler GR : Endovascular occlusion of intracranial aneurysms with electrically detachable coils : correlation of aneurysm neck size and treatment results. AJNR Am J Neuroradiol 15 : 815-820, 1994

4. Gerlach R, Beck J, Setzer M, Vatter H, Berkefeld J, Du Mesnil de Rochemont $\mathrm{R}$, et al. : Treatment related morbidity of unruptured intracranial aneurysms : results of a prospective single centre series with an interdisciplinary approach over a 6 year period (1999-2005). J Neurol Neurosurg Psychiatry 78 : 864-871, 2007

5. Gonzalez N, Sedrak M, Martin N, Vinuela F : Impact of anatomic features in the endovascular embolization of 181 anterior communicating artery aneurysms. Stroke $39: 2776-2782,2008$

6. Hademenos GJ, Massoud TF, Turjman F, Sayre JW : Anatomical and morphological factors correlating with rupture of intracranial aneurysms in patients referred for endovascular treatment. Neuroradiology 40 : 755-760, 1998

7. Hernesniemi J, Dashti R, Lehecka M, Niemelä M, Rinne J, Lehto H, et al. : Microneurosurgical management of anterior communicating artery aneurysms. Surg Neurol 70 : 8-28; discussion 29, 2008

8. Hwang G, Jung C, Sheen SH, Park H, Kang HS, Lee SH, et al. : Two-year follow-up of contrast stasis within the sac in unruptured aneurysm coil embolization : progressive thrombosis or enlargement? AJNR Am J Neuroradiol 31 : 1929-1934, 2010

9. Jeong YG, Jung YT, Kim MS, Eun CK, Jang SH : Size and location of ruptured intracranial aneurysms. J Korean Neurosurg Soc 45 : 11-15, 2009
10. Jo KI, Yeon JY, Kim KH, Jeon P, Kim JS, Hong SC : Predictors of thromboembolism during coil embolization in patients with unruptured intracranial aneurysm. Acta Neurochir (Wien) $155:$ 1101-1106, 2013

11. Kakalis NM, Mitsos AP, Byrne JV, Ventikos Y : The haemodynamics of endovascular aneurysm treatment : a computational modelling approach for estimating the influence of multiple coil deployment. IEEE Trans Med Imaging 27 : 814-824, 2008

12. Kang DH, Hwang YH, Kim YS, Bae GY, Lee SJ : Cognitive outcome and clinically silent thromboembolic events after coiling of asymptomatic unruptured intracranial aneurysms. Neurosurgery 72 : 638-645; discussion 645,2013

13. Kunz M, Bakhshai Y, Zausinger S, Fesl G, Janssen H, Brückmann H, et al. : Interdisciplinary treatment of unruptured intracranial aneurysms : impact of intraprocedural rupture and ischemia in 563 aneurysms. J Neurol 260 : 1304-1313, 2013

14. Loewenstein JE, Gayle SC, Duffis EJ, Prestigiacomo CJ, Gandhi CD : The natural history and treatment options for unruptured intracranial aneurysms. Int J Vasc Med 2012 : 898052, 2012

15. Molyneux AJ, Kerr RS, Yu LM, Clarke M, Sneade M, Yarnold JA, et al. : International subarachnoid aneurysm trial (ISAT) of neurosurgical clipping versus endovascular coiling in 2143 patients with ruptured intracranial aneurysms : a randomised comparison of effects on survival, dependency, seizures, rebleeding, subgroups, and aneurysm occlusion. Lancet 366 : 809-817, 2005

16. Morales HG, Kim M, Vivas EE, Villa-Uriol MC, Larrabide I, Sola T, et al. : How do coil configuration and packing density influence intra-aneurysmal hemodynamics? AJNR Am J Neuroradiol 32 : 1935-1941, 2011

17. Santillan A, Gobin YP, Greenberg ED, Leng LZ, Riina HA, Stieg PE, et al. : Intraprocedural aneurysmal rupture during coil embolization of brain aneurysms : role of balloon-assisted coiling. AJNR Am J Neuroradiol 33 : 2017-2021, 2012

18. Soeda A, Sakai N, Sakai H, Iihara K, Yamada N, Imakita S, et al. : Thromboembolic events associated with Guglielmi detachable coil embolization of asymptomatic cerebral aneurysms : evaluation of 66 consecutive cases with use of diffusion-weighted MR imaging. AJNR Am J Neuroradiol $24: 127-132,2003$

19. UCAS Japan Investigators, Morita A, Kirino T, Hashi K, Aoki N, Fukuhara $S$, et al. : The natural course of unruptured cerebral aneurysms in a Japanese cohort. N Engl J Med 366 : 2474-2482, 2012

20. Uemura A, Kamo M, Matsukawa H : Angiographic outcome after endovascular therapy for anterior communicating artery aneurysms : correlation with vascular morphological features. Jpn J Radiol 30 : 624-627, 2012

21. Vlak MH, Algra A, Brandenburg R, Rinkel GJ : Prevalence of unruptured intracranial aneurysms, with emphasis on sex, age, comorbidity, country, and time period : a systematic review and meta-analysis. Lancet Neurol $10: 626-636,2011$

22. Wiebers DO, Whisnant JP, Huston J 3rd, Meissner I, Brown RD Jr, Piepgras DG, et al. : Unruptured intracranial aneurysms : natural history, clinical outcome, and risks of surgical and endovascular treatment. Lancet 362 : 103-110, 2003

23. Zhang Y, Li G, Cai Y, Zhu J, Huang S, Li T, et al. : Rupture during the endovascular treatment of intracranial aneurysms : outcomes and technical aspects. Acta Neurochir (Wien) $155: 569-577,2013$ 